\title{
Cardiac PET assessment of myocardial microvascular flow may help identify subclinical left ventricular dysfunction and increased risk from aortic stenosis
}

\author{
Ningxin Wan, MD, a and Mark I. Travin, MD, MASNC ${ }^{b}$ \\ a Division of Cardiology, Department of Medicine, Montefiore Medical Center and the Albert \\ Einstein College of Medicine, Bronx, NY \\ b Division of Nuclear Medicine, Department of Radiology, Montefiore Medical Center and the \\ Albert Einstein College of Medicine, Bronx, NY
}

Received May 13, 2019; accepted May 14, 2019

doi: $10.1007 / \mathrm{s} 12350-019-01759-z$

\section{See related article, pp. 579-588}

Diseases of cardiac valves involve not only the valves themselves, but also induce deleterious changes to the cardiac chambers. Aortic valve stenosis, one of the most prevalent valvular diseases in elderly adults, is typically followed by structural alterations in the left ventricle ( $\mathrm{LV})$ that are initially in part an adaptive response attempting to relieve wall stress and maintain cardiac output, but that eventually become maladaptive. ${ }^{1}$ Components of $\mathrm{LV}$ remodeling may persist after valve replacement and contribute to adverse events prior to and potentially even after valve replacement. Better understanding of the processes occurring, and improved methods of identifying them, could help guide timing of interventional therapy and thereby improve patient outcomes both before and after therapeutic interventions.

The most recognized ventricular changes are myocardial hypertrophic responses that include concentric remodeling, concentric hypertrophy, and eccentric hypertrophy. Although these changes are viewed as compensatory efforts to overcome the increased ventricular pressure load, the pattern and severity of LV hypertrophy (LVH) do not strictly

Reprint requests: Mark I. Travin, MD, MASNC, Division of Nuclear Medicine, Department of Radiology, Montefiore Medical Center and the Albert Einstein College of Medicine, 111 E. 210th Street, Bronx, NY10467-2490; mtravin@attglobal.net

J Nucl Cardiol 2021;28:589-93.

$1071-3581 / \$ 34.00$

Copyright (C) 2019 American Society of Nuclear Cardiology. correlate with the severity of valve stenosis as other factors, such as male gender, advanced age, obesity, and genetic factors, and appear to be more significantly associated with the hypertrophy. ${ }^{1}$ Moreover, in aortic stenosis (AS) patients with preserved LV ejection fraction (LVEF), the presence of concentric hypertrophy is independently associated with adverse events including mortality, even after adjustment for the aortic valve area index. $^{2}$ Thus, assessing stenotic severity by itself is insufficient to determine the risk of an adverse outcome.

To better understand mechanisms of hypertrophic LV remodeling in AS, studies have analyzed and compared the two most common models of LV pressure overload: arterial hypertension and AS. Under both conditions, there is a mixture of different patterns of hypertrophic remodeling and a similarly increased prevalence of inappropriate LV mass defined as mass exceeding the value expected for body size and stroke work. However, when LVH is present, AS patients have more severe LV mass excess and a higher prevalence of the concentric geometric pattern. ${ }^{3}$ Apart from differences of morphologic patterns, LV systolic function is also affected differently in the two groups. Compared with hypertensive patients, AS patients have LV dysfunction that is more prevalent and more severe, and that is also independent of LV afterload. These distinct characteristics of AS LVH remodeling must be considered when using image findings to characterize disease severity and guide therapy.

In AS patients, a key turning point from hypertrophy to heart failure appears to be an increase in the rate of myocyte apoptosis that may be a response to the mechanical forces associated with high afterload, but may also be related to an increase in angiotensin II and 
to ischemia. The loss of myocytes leads to fibrosis. ${ }^{1}$ These processes have been demonstrated in AS of varying severity using histopathologic analysis ${ }^{4}$ and by contrast-enhanced cardiac magnetic resonance imaging (MRI), ${ }^{5}$ and correlate with LV systolic dysfunction.

Myocardial fibrosis in AS patients presents in two categories-interstitial and replacement. Interstitial fibrosis is thought to be the reversible phase that occurs prior to irreversible replacement fibrosis. ${ }^{5}$ These types of fibrosis are distinguishable by cardiac MRI, with interstitial fibrosis manifesting as expansion of the extracellular volume detectable by T1 mapping, while replacement fibrosis that accumulates following apoptotic myocyte loss can be quantified by late gadolinium enhancement (LGE). Both forms of fibrosis can progress rapidly. When normal myocardium transitions to interstitial fibrosis, and as replacement fibrosis starts to surface, there is a steady worsening of prognosis. Once replacement fibrosis is detected by LGE, it is irreversible even after aortic valve replacement. ${ }^{6}$ Studies have shown a significant association between both types of myocardial fibrosis and mortality, with or without valve replacement. $^{5}$

Another cardiovascular imaging technique, speckletracking echocardiography, is able to accurately measure deformation of myocardium, thus detecting early, subtle effects of LV functional alterations. The most robust and clinically significant parameter is global longitudinal strain (GLS). As the severity of AS progresses, GLS incrementally deteriorates prior to a decrease in conventional echocardiographically measured LVEF. Reduced GLS is associated with the LV mass index and the aortic valve area, ${ }^{7,8}$ as well as other signs of biomechanical stress in the heart, including left atrial size and the $\mathrm{N}$-terminal pro-brain natriuretic peptide (NT-proBNP). ${ }^{7}$ AS patients with even mildly impaired GLS $(>-18 \%)$ are more likely to progress to the symptomatic stage, thus needing aortic valve replacement. ${ }^{8}$ While aortic valve replacement can improve hemodynamic stress and induces significant reverse remodeling starting in the immediate postoperative period, ${ }^{9}$ patients with severely impaired GLS $(>-$ 9.9\%) tend to show a lack of reverse modeling. ${ }^{10}$ Significantly reduced GLS has been shown to a be strong, independent predictor of all-cause mortality with or without valve replacement. ${ }^{11}$ Overall mortality increases incrementally as GLS worsens. ${ }^{7}$

\section{ABNORMAL MYOCARDIAL BLOOD FLOW IN PATIENTS WITH AORTIC STENOSIS}

An important consequence of AS associated LV remodeling and dysfunction, and likely a key contributor to it, is alterations in coronary myocardial blood flow.
Angina pectoris is a common presentation of severe AS, but only approximately half of those patients have underlying coronary artery disease detectable by conventional coronary angiography. Often the problem involves the microvasculature which can be studied with techniques that assess quantitative coronary blood flow.

Using Doppler echocardiography to quantitate coronary blood flow (CBF) in the left anterior descending (LAD) artery in patients with severe AS but preserved LVEF, and with no coronary stenoses $\geq 50 \%$ on angiography, Meimoun et $a l^{12}$ found coronary flow reserve (CFR) to be significantly lower compared with a cohort of controls, more so for patients with symptoms. Interestingly, while by univariate analysis the indexed LV mass correlated with CFR, by multivariate analysis it was no longer significant, with the only variables independently correlating with CFR being the left ventricular rate-pressure product (LVRPP) that multiples the heart rate by the sum of the systemic systolic pressure and the transvalvular gradient (i.e., the effective ventricular pressure), the pulmonary artery systolic pressure, and the log NT-proBNP, with the latter variable thought to represent a combination of clinical, morphologic, and hemodynamic factors that reflect the high afterload pressure imposed on the LV. Also using echocardiography, Galiuto et $a l^{13}$ assessed CBF by Doppler measurements of LAD flow and by myocardial contrast echocardiographic enhancement of the myocardium. While compared with controls the absolute resting CBF was higher in severe AS with LVH but preserved LVEF and no angiographic CAD, the mass-corrected CBF velocity index (CBFVI) was lower as was the CFR. In addition, from analysis of biopsy samples obtained during surgical valve replacement, CBFVI and CFR inversely correlated with the apoptotic rate, suggesting that ischemia may be an important contributor to the apoptosis.

Alternatively, radionuclide myocardial perfusion imaging (MPI) using positron emission tomography (PET) is now an established, robust method of quantitatively assessing $\mathrm{CBF}$, with demonstrated high diagnostic and prognostic utility in atherosclerotic CAD. This technique is ideally suited to assess how coronary flow, especially in the microvasculature, is affected by AS, and is therefore a potentially useful and convenient noninvasive method to track an important contributor to ventricular injury.

Morphologic studies in AS patients have shown decreased arteriolar density in hypertrophied hearts, termed rarefaction. Nevertheless, despite this lower vessel density, using $\mathrm{H}_{2}^{15} \mathrm{O}$ perfusion PET imaging to separately measure subepicardial and subendocardial myocardial blood flow (MBF), Rajappan et $a l^{14}$ 
demonstrated that in patients with moderate to severe AS who had angiographically normal coronary arteries, resting transmural blood flows were comparable to those in control subjects. At the same time, they found an increasing total resting blood flow proportionate to $\mathrm{LV}$ mass that was attributed to a metabolic vasodilatory response to increased demand. The situation changed with dipyridamole induced vasodilation as transmural blood flow was reduced in AS patients compared with controls, attributed to exhaustion of microcirculatory autoregulatory capacity. In addition, contrary to the resting state, the relationship between $\mathrm{MBF}$ and ventricular mass was lost with hyperemia indicating that the microvascular dysfunction was independent of the degree of myocyte hypertrophy. Given the absence of intramural coronary vessel changes in AS (a distinction from hypertensive $\mathrm{LVH}$ ), vasodilatory reserve impairment was thought likely to be the result of increased extravascular resistance from vessel deformation associated with cardiac mechanical factors. In accord with this reasoning, they found decreased CFR to be related to a higher LVRPP, a higher transvalvular gradient, and a decreased diastolic perfusion time. In this particular study, a valve area of $<0.92 \mathrm{~cm}^{2}$ was the threshold at which subendocardial flow reserve became lower than that for the subepicardium. Subsequent work by this group using mathematical hemodynamic models supported the contention that the valve effective orifice area is the key determinant of CFR, markedly reduced when the area is $<1.0 \mathrm{~cm}^{2}$, and exhausted when the area is $<0.5-0.6 \mathrm{~cm}^{2}$ for which the CFR was calculated to be close to $1 .^{15}$ Such a flow deficiency would expose the myocardium to repetitive ischemia that would result in myocardial loss, such as from the apoptosis described by Galiuto et $a l,{ }^{13}$ and lead to LV dysfunction. Frequent ischemia would cause symptoms, noted to be important in the study by Meimoun et $a l,{ }^{12}$ and would predispose the patient to potentially dangerous arrhythmias and sudden death. Thus, immediate improvement in coronary flow reserve by increasing valve area is an important result of valve replacement, demonstrated in another study by Rajappan et al, ${ }^{16}$ with observations by others that angina and ECG ischemia often quickly resolve while regression of LVH takes much longer. ${ }^{9}$

\section{USING IMAGING TO CHARACTERIZE AND FOLLOW PATIENTS WITH AORTIC STENOSIS}

Given that both echocardiographic assessment of GLS and PET quantitative blood flow (QBF) assessment of microvascular ischemia show promise in identifying preclinical AS induced LV dysfunction, the study by Zhou et $a l^{17}$ in this issue of the Journal of Nuclear
Cardiology proposed to study in more detail how findings from these imaging techniques relate to each other and to disease severity at the valvular level, and how together they could potentially help time the transition from physiological to pathological LV remodeling that increases the likelihood of an adverse outcome. The authors analyzed data from 43 patients who, over a 9-year period, underwent PET MPI $\left({ }^{82} \mathrm{Rb}\right.$ or ${ }^{13} \mathrm{NH}_{3}$ ) for chest pain or dyspnea within 1 year of an echocardiographic study showing trileaflet aortic stenosis of varying severity or aortic sclerosis, all with an LVEF $\geq 50 \%$. There was also a 43-patient cohort of propensity score matched controls. Of note, while only patients with "normal" PET perfusion $($ SSS $<3$ ) were included, coronary angiography was not performed in all patients to definitively exclude obstructive CAD. A key study finding was a stepwise reduction in PET QBF assessed stress flow augmentation, i.e., a decrease in the RPP-corrected global myocardial flow reserve (MFR) with increasing AS severity that, despite a relatively low sample size, achieved statistical significance (although this was mostly from a severe drop in the patients with moderate to severe AS).

Regarding adverse LV effects, with increased valve stenosis severity echocardiographically assessed LV remodeling became increasingly abnormal, and this was associated with a greater than 2-fold higher odds of an abnormal $(<2)$ MFR. In addition, despite no change in LVEF, with increasing AS severity, there were findings of increasing diastolic dysfunction. Finally, with moderate-to-severe AS, there was a worsening of GLS that correlated significantly with MFR and stress myocardial blood flow, possibly indicating that impaired microcirculation contributes to these subtle changes of myocardial function. Importantly, there was no mention of any symptoms or clinical evidence of LV dysfunction in these patients that would have prompted valve replacement.

With regard to patient outcome, over a relatively long ( $\sim 7$ year) follow up period, impaired GLS $(>-$ $18 \%)$ and impaired global MFR $(<2)$ were both significantly and independently associated with an adverse cardiovascular event. An important finding in this study was that whether there was normal or impaired GLS, the presence of impaired MFR dramatically increased the risk of a major adverse cardiac event.

An additional noteworthy observation from the study is the apparent association between aortic sclerosis and reduced corrected MFR compared with controls (although only a trend with $P=.21$ in their Supplemental Figure 3). There was also a significantly worsened average GLS and a suggestion of worsened diastolic dysfunction, both despite no apparent findings of LV 


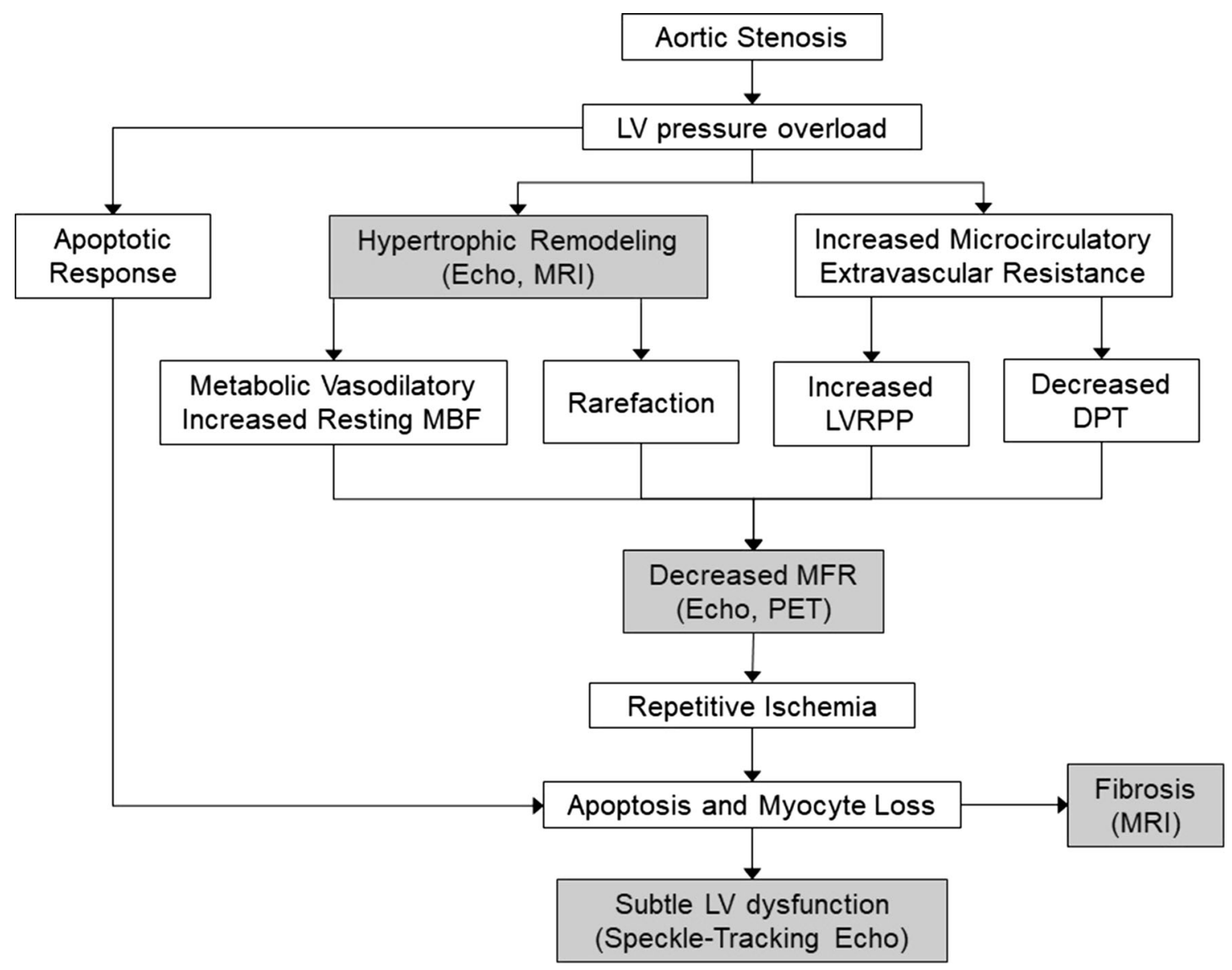

Figure 1. Schematic of left ventricular remodeling and coronary blood flow changes in aortic stenosis. LV pressure overload in AS triggers hypertrophy and apoptosis. Myocyte hypertrophy affects coronary microvasculature by rarefaction (decreased arteriolar density), but increases resting MBF. Increasing stenotic severity increases microcirculatory extravascular resistance via an increase in LVRPP, and decreased DPT. The interplay of these factors impairs MFR. Apoptotic myocyte loss is aggravated by repetitive ischemia and leads to subtle LV dysfunction. Multiple imaging modalities (shaded boxes), including PET, cardiac MRI, and speckle-tracking echocardiography, could be used to help detect irreversible myocardial damage before symptoms or conventionally detected ventricular functional decline. $A S$, Aortic stenosis; $D P T$, diastolic perfusion time; Echo, echocardiography; $G L S$, global longitudinal strain; $L V$, left ventricle; $L V R P P$, left ventricular rate pressure product; $M B F$, myocardial blood flow; $M F R$, myocardial flow reserve; $M R I$, magnetic resonance imaging; PET, positron emission tomography.

remodeling, and of course no reason to suspect increased ventricular cavity pressures that would impede microvascular flow. There is evidence that patients with aortic sclerosis who have not yet developed hemodynamically significant LV outflow obstruction are at increased risk of adverse cardiac events including death, ${ }^{18}$ with a report based on MESA (Multi-Ethnic Study of Atherosclerosis) data suggesting that aortic sclerosis may indicate subclinical atherosclerosis. ${ }^{19}$ While in the manuscript under discussion the authors felt it to be unlikely that any patient had flow limiting CAD, only a minority of them had definitive coronary artery imaging, and thus it is possible that nonobstructive atherosclerotic changes may have affected the microvasculature in some of the patients. For sure, the very small number of patients with aortic sclerosis, 9 out of 86 , limits conclusions about this matter.

\section{FUTURE DIRECTIONS}

Figure 1 summarizes parts of our current understanding of the basic concepts of AS induced LV remodeling. More investigations are needed to increase our understanding of these processes, and how imaging can best visualize and measure them. As acknowledged by Zhou and colleagues, theirs is a single center observational analysis with a relatively small sample 
size. The most clinically significant patient group, i.e., patients with moderate to severe AS, was a relatively small portion of the study sample. Larger, probably multicenter studies with patients having a broad range of AS severity are needed explore how integrating all imaging modalities could improve prognostic prediction, and thereby improve management choice and timing. While such information could potentially help guide existing medical therapies or serve as surrogates for evaluating new pharmacologic or potential device treatments, improved image information would more likely refine criteria for aortic valve replacement (AVR), the ultimate treatment of AS. For example, the ongoing EVOLVED trial (Early Valve Replacement Guided by Biomarkers of LV Decompensation in Asymptomatic Patients With Severe AS) ${ }^{20}$ plans to enroll 1000 patients without guideline indications for AVR (i.e., without symptoms, LVEF decline, or need for other cardiac surgery) to assess whether MRI imaged myocardial fibrosis can assist in deciding for early valve replacement. However, in many instances other imaging tools might be more practical than MRI which has limitations in obese patients, those with devices or renal impairment, or patients unable to cooperate. Speckle-tracking echocardiography is clearly a promising imaging modality here, but it relies on a good quality baseline echocardiogram which may be challenging in many instances. Thus, broadly applicable PET MPI quantitative assessment of coronary blood flow has the potential to be an essential component of multimodality imaging assessment and tracking of AS patients in order to choose the ideal time for valve replacement.

\section{Disclosure} interest.

Ningxin Wan and Mark I. Travin have no conflicts of

\section{References}

1. Dweck MR, Boon NA, Newby DE. Calcific aortic stenosis: A disease of the valve and the myocardium. J Am Coll Cardiol 2012;60:1854-63.

2. Capoulade R, Clavel MA, Le Ven F, Dahou A, Thébault C, Tastet $\mathrm{L}$, et al. Impact of left ventricular remodelling patterns on outcomes in patients with aortic stenosis. Eur Heart J Cardiovasc Imaging 2017;18:1378-87.

3. Mureddu GF, Cioffi G, Stefenelli C, Boccanelli A, de Simone G. Compensatory or inappropriate left ventricular mass in different models of left ventricular pressure overload: Comparison between patients with aortic stenosis and arterial hypertension. J Hypertens 2009;27:642-9.

4. Hein S, Arnon E, Kostin S, Schönburg M, Elsässer A, Polyakova $\mathrm{V}$, et al. Progression from compensated hypertrophy to failure in the pressure-overloaded human heart: Structural deterioration and compensatory mechanisms. Circulation 2003;107:984-91.

5. Chin CW, Everett RJ, Kwiecinski J, Vesey AT, Yeung E, Esson G, et al. Myocardial fibrosis and cardiac decompensation in aortic stenosis. JACC Cardiovasc Imaging 2017;10:1320-33.

6. Everett RJ, Clavel MA, Pibarot P, Dweck MR. Timing of intervention in aortic stenosis: A review of current and future strategies. Heart 2018;104:2067-76.

7. Dahl JS, Videbæk L, Poulsen MK, Rudbæk TR, Pellikka PA, Møller JE. Global strain in severe aortic valve stenosis: Relation to clinical outcome after aortic valve replacement. Circ Cardiovasc Imaging 2012;5:613-20.

8. Vollema EM, Sugimoto T, Shen M, Tastet L, Ng AC, Abou R, Marsan NA, et al. Association of left ventricular global longitudinal strain with asymptomatic severe aortic stenosis: Natural course and prognostic value. JAMA Cardiol 2018;3:839-47.

9. Monrad ES, Hess OM, Murakami T, Nonogi H, Corin WJ, Krayenbuehl HP. Time course of regression of left ventricular hypertrophy after aortic valve replacement. Circulation 1988;77:1345-55.

10. Gelsomino S, Luca F, Parise O, Lorusso R, Rao CM, Vizzardi E, et al. Longitudinal strain predicts left ventricular mass regression after aortic valve replacement for severe aortic stenosis and preserved left ventricular function. Heart Vessels 2013;28:775-84.

11. Kusunose K, Goodman A, Parikh R, Barr T, Agarwal S, Popovic $\mathrm{ZB}$, et al. Incremental prognostic value of left ventricular global longitudinal strain in patients with aortic stenosis and preserved ejection fraction. Circ Cardiovasc Imaging 2014;7:938-45.

12. Meimoun P, Germain AL, Elmkies F, Benali T, Boulanger J, Espanel C, Clerc J, Zemir H, Luycx-Bore A, Tribouilloy C. Factors associated with noninvasive coronary flow reserve in severe aortic stenosis. J Am Soc Echocardiogr 2012;25:835-41.

13. Galiuto L, Lotrionte M, Crea F, Anselmi A, Biondi-Zoccai GG, De Giorgio F, et al. Impaired coronary and myocardial flow in severe aortic stenosis is associated with increased apoptosis: A transthoracic Doppler and myocardial contrast echocardiography study. Heart 2006;92:208-12.

14. Rajappan K, Rimoldi OE, Dutka DP, Ariff B, Pennell DJ, Sheridan DJ, et al. Mechanisms of coronary microcirculatory dysfunction in patients with aortic stenosis and angiographically normal coronary arteries. Circulation 2002;105:470-6.

15. Garcia D, Camici PG, Durand L, Rajappan K, Gaillard E, Rimoldi $\mathrm{OE}$, et al. Impairment of coronary flow reserve in aortic stenosis. $\mathrm{J}$ Appl Physiol 2009;106:113-21.

16. Rajappan K, Rimoldi OE, Camici PG, Bellenger NG, Pennell DJ, Sheridan DJ. Functional changes in coronary microcirculation after valve replacement in patients with aortic stenosis. Circulation 2003; 107:3170-5.

17. Zhou W, Bajaj N, Gupta A, Sun Y, Divakaran S, Bibbo C, et al. Coronary microvascular dysfunction, left ventricular remodeling, and clinical outcomes in aortic stenosis. J Nucl Cardiol 2019. h ttps://doi.org/10.1007/s12350-019-01706-y.

18. Otto CM, Lind BK, Kitzman DW, Gersh B, Siscovick DS, for the Cardiovascular Health Study. Association of aortic-valve sclerosis with cardiovascular mortality and morbidity in the elderly. N Engl J Med 1999;341:142-7.

19. Owens DS, Budoff MJ, Katz R, Takasu J, Shavelle DM, Carr JJ, et al. Aortic valve calcium independently predicts coronary and cardiovascular events in a primary prevention population. J Am Coll Cardiol Imaging 2012;5:619-25.

20. https://clinicaltrials.gov/ct2/show/NCT03094143. Accessed 12 May 2019.

Publisher's Note Springer Nature remains neutral with regard to jurisdictional claims in published maps and institutional affiliations. 